\title{
Blind-eye cannulation as a new method for ERCP training: Can we do more than merely teach?
}

\section{(ㄷ)(1) $\ominus$}

\author{
Authors \\ Institutions \\ 1 Digestive Endoscopy Unit, Fondazione Policlinico \\ Universitario Agostino Gemelli IRCSS, Rome, Italy \\ 2 Università Cattolica del Sacro Cuore di Roma, Center for \\ Endoscopi Research Therapeutics and Training (CERTT), \\ Rome, Italy \\ 3 University College London Hospitals, London, UK
}

Ivo Boškoski ${ }^{1,2}$, George Webster ${ }^{3}$, Andrea Tringali ${ }^{1,2}$, Pietro Familiari ${ }^{1,2}$, Vincenzo Perri ${ }^{1,2}$, Guido Costamagna ${ }^{1,2}$

submitted 12.9.2019

accepted after revision 14.10.2019

\author{
Bibliography \\ DOI https://doi.org/10.1055/a-1066-8880 | \\ Endoscopy International Open 2020; 08: E186-E188 \\ (c) Georg Thieme Verlag KG Stuttgart · New York \\ elSSN 2196-9736
}

Corresponding author

Ivo Boškoski, MD, PhD, Fondazione Policlinico Universitario Agostino Gemelli IRCSS, Largo A. Gemelli, 800168 Rome, Italy

Fax: + 39-06-30156580

ivo.boskoski@policlinicogemelli.it

\section{ABSTRACT}

Background and study aims Endoscopic retrograde cholangiopancreatography (ERCP) requires extensive hands-on training. Currently in ERCP training there are two very important problems that need attention: lack of standardized commands for communication, and misunderstanding between the trainee and the trainer.

Methods A method of "blind-eye" cannulation was developed with two paired trainees using the Boškoski-Costamagna ERCP Trainer. The trainee who was holding the duodenoscope was blind-folded and the other trainee gave instructions on what maneuvers were necessary to achieve cannulation, under the supervision of a trainer. Before starting cannulation, a standard list of commands was agreed by the operators.

Results The blind-eye method of cannulation teaches the operator trainee to listen and the assistant trainee to teach. Trainees use standardized commands. During the training session the two trainees swapped roles.

Conclusions Currently, we do not have proof that this type of training is useful, therefore, scientific studies are needed for confirmation. However, we believe that this ERCP model may provide an entirely safe means of improving communication and technical proficiency.
Endoscopic retrograde cholangiopancreatography (ERCP) is among the most technically demanding and high-risk procedures in gastrointestinal endoscopy. ERCP is currently taught through supervised hands-on training on patients, mostly due to the lack of training programs for novices or absence of structured training on simulators [1]. The advent of simulators for ERCP is slowly changing the training process. In many countries preclinical simulator training is obligatory before performing procedures on patients. With that, trainees gain sufficient hands-on training without compromising patient safety and further progression is done in a safe learning environment [2]. Today there are several different and useful platforms for ERCP training, however, limited data are available on the impact of simulators in ERCP training [3-9]. Currently, there are two very important but still poorly addressed problems that need attention. The first one is lack of standardized commands for communication between the trainee and the trainer. The second is that trainees may not adequately listen to, understand or respond to what the trainer is suggesting/requiring. Misunderstanding between a trainee and a trainer could lead to poor outcome or complications. Correct communication between trainee and trainer is therefore essential. This problem may be overcome by agreeing to a list of "commands" prior to a trainee commencing a procedure. Better still would be gaining agreement on validated commands within the ERCP training community to be used during structured ERCP training.

A fundamental of ERCP training is that both trainee and trainer "speak the same language." It is vital that the action expected from a particular command is understood by both parties before training procedures are undertaken. In $>$ Table 1 we propose some examples of the commands. There can be some variation around these, but absolute clarity is essential. The second problem is more difficult to solve. The great majority of ERCP training has traditionally been on patients, and a com- 
- Table 1 Examples of proposed commands to be used during ERCP training.

\begin{tabular}{|c|c|}
\hline Command & Meaning \\
\hline Straight & Straighten the scope \\
\hline $\begin{array}{l}\text { “Tip up" or "big } \\
\text { wheel up" }\end{array}$ & Bring the scope tip towards the papilla \\
\hline $\begin{array}{l}\text { “Tip down” or big } \\
\text { wheel down }\end{array}$ & Bring the scope tip away from the papilla \\
\hline "Push out" & $\begin{array}{l}\text { Push the accessory out from the scope } \\
\text { towards the papilla }\end{array}$ \\
\hline "Pull in" & $\begin{array}{l}\text { Pull the accessory into the scope, far from } \\
\text { the papilla }\end{array}$ \\
\hline Open the elevator & Bridge down \\
\hline Close the elevator & Bridge up \\
\hline Scope in & Push the scope into the patient \\
\hline Scope back & Pull the scope out \\
\hline Turn left & Refers to rotating small wheel \\
\hline Turn right & Refers to rotating small wheel \\
\hline Body/wrist joint left & Is different from moving the small wheel \\
\hline Body/wrist joint right & Is different from moving the small wheel \\
\hline
\end{tabular}

bination of trainee anxiety about succeeding immediately and time pressure may lead to trainees responding to an instruction to make a maneuver (for example, to "turn the small wheel to the left") by doing exactly the opposite. This may have important clinical implications (e.g. procedure failure or complications). A third challenge is that trainers may be highly skilled at performing ERCP themselves ('unconsciously competent') but poor at verbalizing to trainees the maneuvers necessary to achieve an optimal result. These challenges may be effectively addressed with the Boškoski-Costamagna ERCP trainer (Cook Medical, Limerick Ireland) ( $\triangleright$ Fig. 1). This ERCP trainer is capable of reproducing all maneuvers in ERCP, from basic to very advanced, including sphincterotomy and pre-cut, and is also equipped with simulated fluoroscopy. The effect of scope/ wheel maneuvers relative to the ampulla can also be directly viewed ( $>$ Fig. 2). It has recently been validated for cannulation as well as for sphincterotomy by the Rotterdam group in two different studies $[10,11]$.

To improve trainee-trainer communication (including trainees' response to instruction, and trainers' clear verbalizing of what is needed) we developed the method of "blind-eye" cannulation. In this training scenario, two trainees are paired. The trainee who is holding the duodenoscope is blind-folded and the other trainee gives instructions on what maneuvers are necessary to achieve cannulation. All is done under the supervision of a trainer. Before starting cannulation, a standard list of commands is agreed upon between the operators.

Blind-eye cannulation starts with the scope in front of the papilla and the sphincterotome inside the scope, just behind the elevator. On a signal from the supervising trainer, the can-

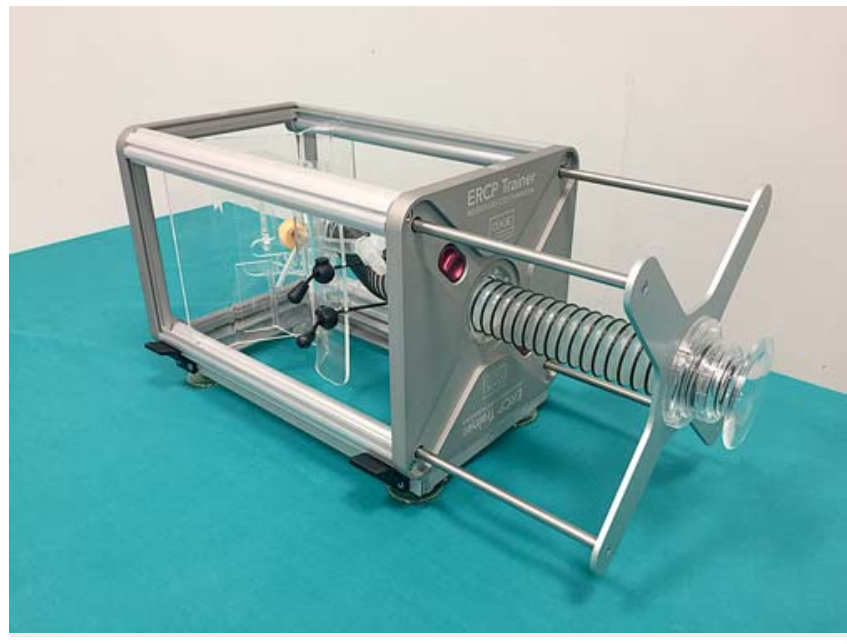

- Fig. 1 The Boškoksi-Costamagna ERCP Trainer (Cook Medical, Limerick Ireland).

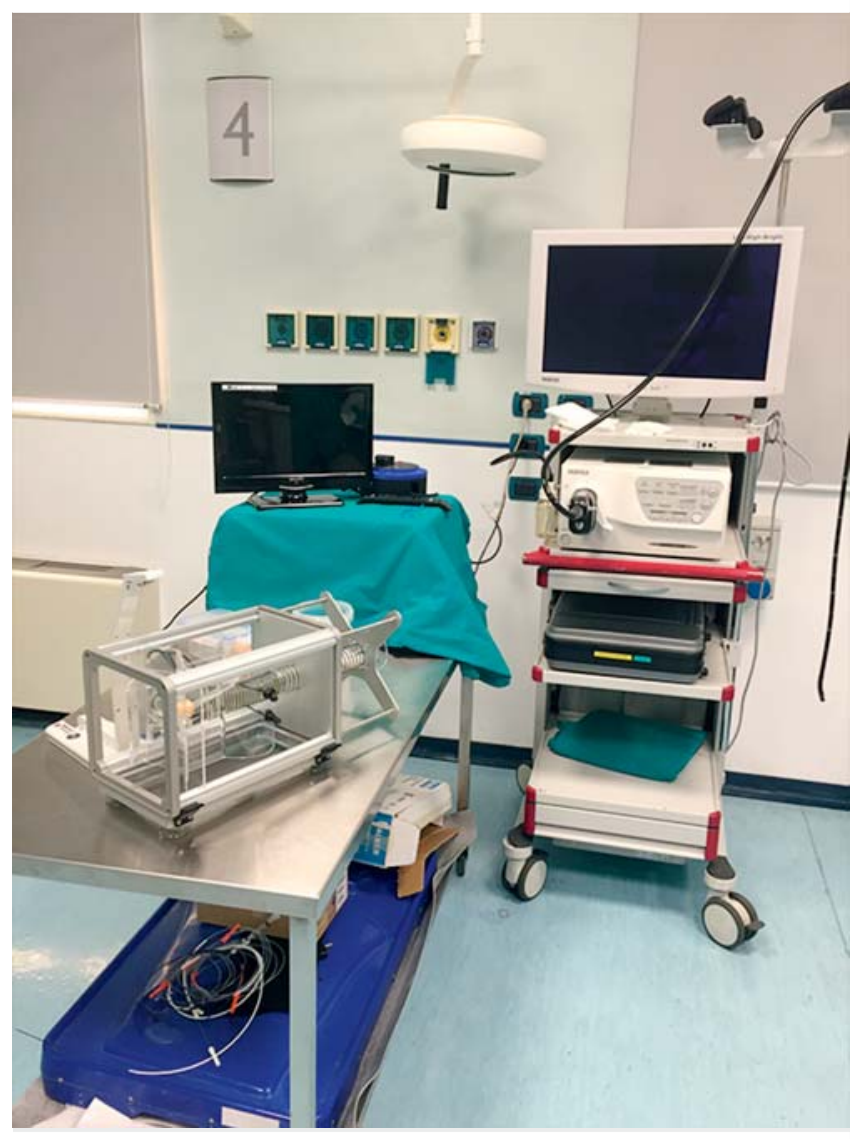

- Fig. 2 Standard setting of the ERCP Trainer with simulated fluoroscopy (camera on the top of the ERCP Trainer).

nulation starts. In this way the operator trainee learns to listen and the assistant trainee to teach. During the training session, the two trainees swap roles ( $>$ Fig. 3 ). We perform blind-eye cannulation at the end of every ERCP training sessions on the ERCP Trainer and it is always supervised by expert trainers. The blind-folded trainee learns to follow instruction and the assis- 


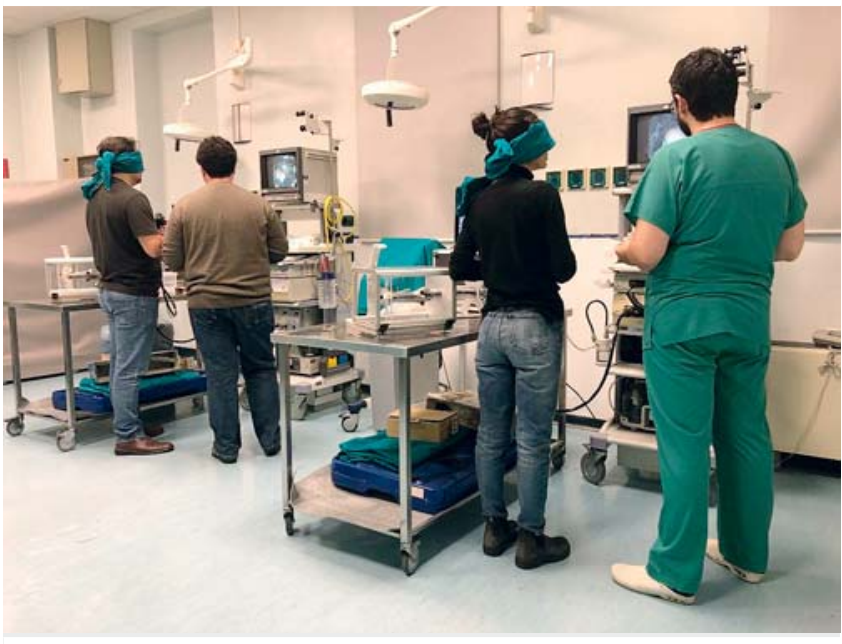

- Fig. 3 Blind-eye cannulation for ERCP training.

tant trainee acquires a better understanding of the techniques needed to achieve cannulation. In addition, to the best of our knowledge, this is the first report of an eyes-blinded type of training for all medical and non-medical sciences.

\section{Conclusion}

Currently, we do not have proof that this type of training is useful. Therefore, scientific studies are needed to confirm that. However, we believe that this ERCP model may provide an entirely safe means of improving communication and technical proficiency, so that training in ERCP does not involve the "blind guiding the blind"!

\section{Competing interests}

Professor Costamagna is a consultant for Olympus, Cook Medical, and Boston Scientific. Dr. Webster has received honoraria for Advisory Boards and lecturing on behalf of Cook
Medical, Boston Scientific, and Pentax Medical. Dr. Boškoski is a research grant holder from Apollo Endosurgery and a consultant for Apollo Endosurgery, Cook Medical, and Boston Scientific. Dr. Tringali is a consultant for Boston Scientific.

References

[1] Jirapinyo PI, Thompson CC. Current status of endoscopic simulation in gastroenterology fellowship training programs. Surg Endosc 2015; 29: 1913-1919

[2] van der Wiel SE, Küttner Magalhães RR, Rocha Gonçalves CR et al. Simulator training in gastrointestinal endoscopy - From basic training to advanced endoscopic procedures. Best Pract Res Clin Gastroenterol 2016; 30: 375-387

[3] Frimberger E, von Delius S, Rösch T. A novel and practicable ERCP training system with simulated fluoroscopy. Endoscopy 2008; 40: 517-520

[4] Neumann M, Mayer G, Ell C et al. The Erlangen Endo-Trainer: life-like simulation for diagnostic and interventional endoscopic retrograde cholangiography. Endoscopy 2000; 32: 906-910

[5] Matthes K, Cohen J. The Neo-Papilla: a new modification of porcine ex vivo simulators for ERCP training (with videos). Gastrointest EndosC 2006; 64: 570-576

[6] Bar-Meir S. Simbionix simulator. Gastrointest Endosc Clin N Am 2006; 16: 471-478, vii

[7] Leung JW, Lee JG, Rojany M et al. Development of a novel ERCP mechanical simulator. Gastrointest Endosc 2007; 65: 1056-1062

[8] von Delius S, Thies P, Meining A et al. Validation of the X-Vision ERCP Training System and technical challenges during early training of sphincterotomy. Clin Gastroenterol Hepatol 2009; 7: 389-396

[9] Itoi T, Gotoda T, Baron TH et al. Creation of simulated papillae for endoscopic sphincterotomy and papillectomy training by using in vivo and ex vivo pig model. Gastrointestinal Endoscopy 2013; 77: 793-800

[10] van der Wiel SE, Koch AD, Bruno MJ. Face and construct validity of a novel mechanical ERCP simulator. Endosc Int Open 2018; 6: E758E765

[11] van der Wiel SE, Koch AD, Bruno MJ. Face validity of a synthetic papilla designed for biliary sphincterotomy training. Endosc Int Open 2019; 7: E757-E761 